\title{
Orthogonal polarization approach for three dimensional georadar surveys.
}

\author{
Maurizio Lualdi ${ }^{*}$, Federico Lombardi
}

Politecnico of Milan, Piazza Leonardo da Vinci 32, 20133 Milan, Italy

* Corresponding author. Tel.: +390223994226, Fax: +39 0223994220, email: maurizio.lualdi@polimi.it

\begin{abstract}
This paper presents the results obtained from the combination of co-pole GPR data collected along perpendicular directions. The scope is to demonstrate how this approach can efficiently overcome pitfalls of traditional single orientation surveys and ensure target detection regardless their geometrical and physical properties. This is of highly importance especially when acquiring across targets that show directional dependencies of the preferential scattering components. The work relies on four field examples, each of them illustrating in details the improvements and the advantages a single image resulting from the stack of the two volumes can show, in particular for what concern target imaging.
\end{abstract}

Keywords: Ground penetrating radar, polarization, 3D surveys, structures inspection.

\section{Introduction}

Ground penetrating radar technique has proven to be a practical and productive method for non-destructive diagnosis of shallow subsurface ([1], [2], [3]). Commonly, GPR surveys are performed along single bidimensional profiles or sparsely sampled grids. Nevertheless a faster data collection, 2D profiles could lead to incorrect reconstruction of subsurface features, especially when geometry of the investigated targets is complex. 
Three dimensional acquisitions are more time consuming and expensive than a bidimensional ones ([4]) because it is necessary to acquire a dense and regular grid of traces, with a sample spacing sufficient to prevent spatial aliasing problems ([5]). Fulfilling these constraints guarantees a fully reconstruction of the geometry of any targets. Specific problems that need a 3D approach to be solved are, for example, linear targets ([6], [7], [8]), fault and geological features ([9], [10], [11], [12]), archaeology ([13], [14], [15]), cultural heritage ([16], [17], [18]) and UXO detection ([19], [20], [21]).

Major concerns about georadar capabilities are related to the directional sensibility of the EM wavefield. Most GPR systems employ linearly polarized dipole antennae with transmitter emitting an electric field polarized parallel to the long axis of the dipole and a receiver that records only the component parallel to its long axis ([22]). However, it has been noticed that various targets of georadar surveys, such as buried pipes and fractures, have strong polarization dependent scattering characteristics ([23], [24], [25], [26]). Numerous studies have exploited and investigated these features and their relationship with radar imaging (e.g. [7], [27], [28]), showing that to map any subsurface target it is necessary to perform a 3D full polarization georadar survey. In [29], authors presented the possibility of summing georadar data acquired along perpendicular directions to improve target detection. A work from the authors ([30]) demonstrated that the combination of data acquired with through a couple of dipoles oriented perpendicular to each other ensures linear target detection regardless relative geometry between transmitters and targets and physical properties of the target. This work demonstrates the effectiveness of the proposed approach in 3D ground penetrating radar applications. Further considerations are focused on final images 
processing, as the stack process can bring an amplitude range that might masks weaker targets.

\section{Surveys description and results}

The four 3D GPR experiments were all recorded using the Aladdin georadar system (by IDS - Ingegneria dei Sistemi, Italy), which consists in a couple of two $2 \mathrm{GHz}$ dipole antennae (with offset of $6 \mathrm{~cm}$ for both configuration) at orthogonal polarization, and the positioning system PSG (Pad System for Georadar, U.S. Patent no. US 7,199,748 B2 of Politecnico di Milano, Italy, see [31]). The device used for the presented field experiments and its design is illustrated in Fig. 1.

\section{Figure 1}

This configuration guarantees precise matching between the two CMP of the parallel (VV) and perpendicular (HH) orientation, in respect to the survey direction, permitting joint orthogonally polarized scans to be acquired in a single pass. Accurate profile spacing was obtained through PSG, a pad whose surface is modelled with parallel tracks that are few millimetres high. The GPR antenna is dragged along the tracks so that parallel and regularly spaced profiles are rapidly executed without varying antenna orientation during the whole survey.

The two analysed stacking strategies were the arithmetic mean of the raw data and of the processed ones. Data were processed using a tool developed by Politecnico di Milano running on Mathworks MATLAB software.

Radar images are shown through depth slices, obtained by plotting the amplitude of the brightest reflector over the specified depth range. Further on, all the presented images are displayed with the same amplitude range and contrast settings so the amplitude 
response of each component can be compared.

\subsection{Palazzo Pisani, Venice, Italy.}

First survey was carried out in Venice to investigate the geometry of local structural metallic features, so called "fiube"; these elements were used to connect the façade of a building to the floors. The only aid for detecting such targets is the presence of the end of a "fiuba" on the façade (Fig. 2a). Acquisition was performed on the floor (Fig. 2b, the white arrow represents survey direction and starting profile) and parameters for both configuration are given in Table 1.

\section{Table 1}

To obtain a square mesh, data were interpolated to a $0.8 \mathrm{~cm}$ step-increment grid.

\section{Figure 2}

Processing consisted in five steps ([5]), described in Table 2.

\section{Table 2}

Raw stack was computed after the alignment process, while the processed one after data envelope.

Images from single azimuth processing are shown in Fig. 3a (HH configuration) and Fig. 3b (VV configuration). Two "fiube" are detected (see sketched representation of targets in Fig. 4) and the comparison shows the different sensitivity of the antenna orientation to linear targets. Target oriented perpendicular to the survey direction (target marked A in Fig. 4) is clearly visible in the $\mathrm{HH}$ acquisition (Fig. 3a), while almost invisible in the VV one (Fig. 3b, except for a 3D scattering effect at the end of the bar). Concerning the inclined fiuba (target B in Fig. 4), its representation is visible in both configurations with a lower response. 
$97 \quad$ Figure 3

\section{$98 \quad$ Figure 4}

99 Final images coming from the azimuthal stack are presented in Fig. 3c (raw) and Fig. 3d

100 (processed).

101 As can be seen, results confirm what was expected, that is a precise reconstruction of targets regardless their orientation.

There are no noticeable differences between the two results, except that Fig. 3d (envelope stack) is a little more degraded, as a consequence of the higher noise of the HH image (Fig 3a). This aspect is related to the difference in antenna pattern between 106 the two configurations.

107 For this reason, a stacking strategy based on pixels amplitude comparison was computed. The concept is the following: if a target is clearly visible there is no need to adding up the complementary polarization contribute. Starting from the computation of the absolute value of the amplitude difference between corresponding pixels, a threshold value is chosen to set if these pixels should be included in the algorithm or not. Only the couples whose absolute difference is less than or equal to the threshold are stacked, while the maximum of the two pixels is taken if their difference exceeds it. In case of degraded data, this approach averages and lowers noisy regions of the image, as noise is less sensitive to wave polarization. For linear targets, for which antenna orientation has a strong impact, this scheme ensures that the optimum condition will always be

117 selected. These features highly improve the signal to noise ratio and, consequently, image resolution. Another benefit is a better target shape reconstruction. The threshold value is varied to take into account the amount of pixels that will be stacked. Fig. 5 represent the final image obtained applying a threshold starting from a 
121 value of 0 (the maximum values are always taken) up to 0.7 (close to the average of the

122 entire images).

\section{Figure 5}

124 If one consider a threshold of 0.3 (Fig. 5d), that means that pixels are stacked if and 125 only if their difference in amplitude is less than or equal to 0.3 , the following 126 considerations can be made:

- Noise is highly mitigated, compared to the HH image (Fig. 3a).

- Inclined target is represented with a better resolution and higher intensity, compared to the VV image (Fig. 3b) and the stack of the raw data (Fig. 3c). essential differences between the two techniques, is the possibility to easily manage the amplitude range of the final image and the amount of noise that can occur.

Another example, taken from a survey on the Gaetano Donizetti Theatre in Bergamo (Fig. 6a), was aimed to detect the presence of metallic supports to the letters of the marble inscription on top of the façade (Fig. 6b).

\section{Figure 6}

139 All profiles were acquired oriented from the top of the façade to the ground (black 140 arrow in Fig. 6b).

141 Acquisition parameters are listed in Table 3.

\section{Table 3}

143 As before, a 0.8 spacing square mesh was created. Processing scheme, detailed in Table 144 4, included also a background removal after the traces alignment, in order to reduce the 
effect of the marble slabs.

\section{Table 4}

147 Raw data stack was computed before the background removal step.

148 Fig. 7a and Fig. 7b describe single azimuth results (HH and VV, respectively).

\section{$149 \quad$ Figure 7}

\section{Figure 8}

151 Some remains of the slabs are still present (see the vertical and inclined sticks of the N 152 letter), but the anchorage system of the inscription is clearly identified. Referring to the 153 sketch in Fig. 8, two of the three bars (targets A and B) are oriented crossline to the 154 survey line, while the other one (target C) is parallel to it. The azimuthal stack presented in Fig. 7c (raw) and Fig. 7d (processed) demonstrates the benefits of displaying all linear targets independently from their orientation in a single image. Comparing the two figures, one can see that stack of the enveloped data (fig. 7d) produce a more clean and focused image, in particular nearby the vertical oriented bar.

159 Further on, the stack highlights the curved shape of the bar B marked in Fig. 8 with a 160 dotted circle. This aspect does not appear in the HH image (Fig. 7a), while in the VV one (Fig. $7 b$ ) there are some traces of the stroke oriented nearly parallel to the antenna.

162 Essentially, this feature is hardly detectable looking only through single azimuth volume. The amplitude related stack (Fig. 9), following the considerations made

164 previously in Subsec. 2.1, shows improvements in decreasing the remnants of the 165 background signal and reconstruction of the three metallic supports.

\section{Figure 9}

167 In this case, best results are obtained with a threshold value around 0.3-0.4 (Fig. 9d 9e). 


\subsection{Underfloor heating system, Milan, Italy.}

171 A buried heating coil was investigated to analyse the effect of polarization on water

172 filled plastic pipes. In Fig. 10 is pictured the acquired area before cement application,

173 showing the presence pipes of different length, orientation and path. The white arrow in

174 Fig. 10 represents survey geometry and the first acquired profile.

175 Figure 10

176 Table 5 describes survey parameters and data volume details. Last profile ( $\left.\mathrm{n}^{\circ} 113\right)$ was

177 acquired near the wall (marked in Fig. 10).

\section{Table 5}

179 The standard processing flow, reported in Table 6, was applied on the acquired profiles.

\section{Table 6}

181 As for the other experiments, raw stack consisted in the arithmetic mean of the two datasets after traces alignment and the processed one after data envelope.

183 The single azimuth results are pictured in Fig. 11a (HH) and 11b (VV).

\section{Figure 11}

185 From a first analysis, there are visible amplitude differences between the HH image 186 (Fig. 11a) and the VV (Fig. 11b) one, with the last leading on the first. This effect is related to the response of conductive targets depending not only on their geometry but also on their length ([22]). Fig. 10 shows that pipes oriented along the survey direction

189 are longer than the others, nearly twice, and so their intensity is almost doubled. The chessboard surrounding pipes is the grid in which they are cast, which is at the same depth and generates a quite homogeneous scattering. Another detail visible in Fig. 11a and 11b is a second pipes mesh just aside of the 
regular one. This effect is due to the proximity of the targets, the tails of which hyperbola intersecting each other create (feature highlighted in Fig. 12a and 12b) a shifted and delayed version of the real targets. Interpretation and reconstruction are provided in Fig. 12c and 12d.

\section{Figure 12}

The two dipoles orientations are not able to follow the curved shape of the pipes, as in Subsec. 2.2.

Multiazimuth pictures are shown in figures 11c (raw) and 11d (processed). Stack of the processed data provides a better results, in terms of target continuity and definition (pipes are fully reconstructed), but also enhances the effect of the floor grid. Raw data stack mitigates its response but lose some parts of the target, especially close to the turning points. The same considerations can be made for the synthetic mesh: combining processed data (Fig. 11d) the hyperbola interference effect increases, as the stack does not differentiate it from the real pipes. The stack of the raw data (Fig. 11c) instead has a mitigation effect, due to the arithmetic mean. Analysing the threshold stack (Fig. 13), it is clear that one could obtain an optimum results (e.g. with a threshold value of 0.4, Fig. 13e). Differences in the effect of the threshold are evident.

\section{Figure 13}

The threshold effect is a noise reduction and an enhancement of the pipes, improving their interpretability. Another benefit is the lowering of the tails-generated mesh. These considerations are in agreement with what was explained in Subsec. 2.1.

\subsection{Wall inspection, Milan, Italy.}

In this case, differences in imaging are due to variations in dielectric properties between 
217 bricks and lime mortar. However, they are not as evident as for conductive linear

218 targets.

219 Dataset consists in a volume of profiles acquired on a common bricks wall (geometry

220 shown in Fig. 14a and 14b), with parameters detailed in Table 7.

\section{$221 \quad$ Table 7}

222 As in Subsec. 2.2, background removal was applied to reduce the impact of plastering process (processing described in Table 8). Further on, a data windowing was computed. Raw stack was computed before this step, while processed one after the envelope

225 display.

\section{Table 8}

227 Single azimuth depth slices are presented in figures $14 \mathrm{a}(\mathrm{HH})$ and $14 \mathrm{~b}(\mathrm{VV})$.

228 Differences in details imaging are clear, for the HH (Fig. 14a) configuration better depicts horizontal segmentations of the wall, losing details of the vertical mortar lime. Vertical texture is better identifiable in the VV (Fig. 14b) image. MultiAzimuth stack provides images (Fig. 14c and Fig. 14d) that show some improvements in geometrical reconstructions of the bricks and mortar lime sequence. No great differences are visible between the two stacking techniques, except for the middle region of the image where the raw stack (Fig. 14c) better reconstruct wall texture.

\section{Figure 14}

236 Useful tools to highlight abruptly gray-scale value or colour changes from one pixel to 237 the next are the directional filters, based on the discrete gradient of the image intensity function ([33]). A second order method, Laplacian operator, has been applied on the radar slices to enhance details and sharpness. 
241 Effect of azimuthal stack is clearer (Fig. 15c and 15d), in particular if one looks in the

242 middle and right part of the picture.

\section{$243 \quad$ Figure 15}

244 Single polarization is not able to detect the mortar lime, and so the presence of a brick,

245 while the MultiAzimuth technique permits to obtain a more detailed map of the walls

246 structure.

247 The results show that the combination of MultiAzimuth strategies and image processing

248 technique could resolve complex situation, where the focus is enhancing geometrical

249 texture and features.

\section{Discussion}

252

The potential of a co-pole 3D multi polarization approach for overcoming difficulties belonging to geometry and polarization has been evaluated and demonstrated with several field surveys. In particular, acquisitions have made clear the advantages brought by the combination of the orthogonal polarization images into a single one, without any loss of attributes and resolution. These advantages lie in the opportunity of having a single image with all buried and detected targets, feature that in a field of application characterized by linear target (therefore ruled by polarization theory) is remarkably a surplus value. Considering the two analysed stacking technique, the field surveys have not shown great difference in stacking raw data and processed ones, leaving the choice to the end user and survey settings. What is to be underlined is a remarkable details augmentation and interpretation facility of the resulting images. The risk of overwhelming weaker target reflections in the final image, due to an excessive large amplitude range, has been overcame performing a stack based on pixel 
differences, that mitigates the effect of higher amplitude and at the same time decreases noise level. Computationally, the algorithm performs only a pixel by pixels analysis and a comparison.

This method has revealed its potentiality in civil diagnosis and could be a useful tool for seismic structural assessment ([34], [35]).

\section{Conclusion}

271 As stated at the beginning of the paper, 3D georadar surveys lie on a precise traces

272 positioning, both in crossline and inline direction. The system used in the present work ensures this feature because dipoles emit simultaneously and receive from the same CMP. Obviously, in large areas acquisition antenna arrays are commonly employed ([36]), and so the problem of traces regularity and parallelism between adjacent profiles can originate from the design of the array. In addition, positioning devices such as GPS or Total Station introduce their intrinsic errors together with cumulative ones that must be taken into account when acquiring long profiles ([37], [38]). So, further studies and developments should have to explore the influence that a misplacement of traces and irregular geometry have on the final migrated images ([39]). Secondly, the unanswered question of how many azimuths are needed to ensures that no features might be lost. Because of the time consuming of adding a survey direction, information on its impact is highly necessary for planning a comprehensive experiment without any risk of losing details of the subsurface. The importance of this issue gains more and more weight in case of strongly directional events.

\section{Acknowledgment}

287 The authors are thankful to IDS Company for the supply of georadar equipment. 


\section{References}

290 [1]. Daniels DJ. Ground Penetrating Radar. 2nd ed. London, U.K.: Peter Peregrinus 291 Ltd., 2004.

292

293

294

295

296

297

298

299

300

301

302

303

304

305

306

307

[2]. Jol HM editor. Ground Penetrating Radar: Theory and Applications. 1st ed. Amsterdam, The Netherlands: Elsevier; 2009.

[3]. American Society for Testing and Materials (US). Standard guide for selecting surface geophysical methods, Designation D-6429, Philadelphia, PA; 1999.

[4]. Lualdi M, Binda L, Zanzi L. Acquisition and processing requirements for high quality 3D reconstructions from GPR investigations. Proceedings of the International Symposium Non Destructive Testing in Civil Engineering NDTCE; 2003 Sept. 16-19; Berlin, Germany.

[5]. Yilmaz O. Seismic Data Analysis. 2nd Edition. Tulsa, USA: Society of Exploration Geophysicists; 2001.

[6]. Van der Kruk J, Wapenaar CPA, Fokkema JT, Van den Berg, P.M. Threedimensional imaging of multicomponent ground-penetrating radar data. Geophysics 2003; 68(4): 1241-54.

[7]. Roberts R, Cist D, Kathage A. Full-Resolution GPR imaging applied to utility surveying: Insight from multi-polarization data obtained over a test pit, IWAGPR; 2009 May 27-29; Granada, Spain, p. 126-31. 
[8]. Bernstein R, Oristaglio M, Miller DE, Haldorsen J. Imaging radar maps underground objects in 3-D. IEEE Computer Applications in Power 2000; 13(3): $20-4$

[9]. Grasmueck M, Weger R. 3D GPR reveals complex internal structure of Pleistocene oolitic sandbar. The Leading Edge 2002; 21(7): 634-39.

[10]. Lualdi M, Zanzi L. 2D and 3D experiments to explore the potential benefit of GPR investigations in planning the mining activity of a limestone quarry. Proceedings of the Tenth International Conference Ground Penetrating Radar (GPR 2004) June 21-24; Delft, The Netherlands, p. 613-6.

[11]. Vanneste K, Verbeek K, Petermans T. Pseudo 3D imaging of a slow-slip-rate, active normal fault using shallow geophysical methods: The Geleen fault in the Belgian Maas River valley. Geophysics 2008; 73(1): B1-B9.

[12]. Carpentier SFA, Green AG, Langridge RM, Boschetti S, Doetsch J, Abächerli AN, et al. Flower structures and Riedel shears at a stepover zone along the Alpine Fault (New Zealand) inferred from 2D and 3D GPR images. J. Geopys. Res. 2012; 117: B2.

[13]. Lorenzo H, Novo A, Rial FI, Solla M. Three-dimensional Ground-penetrating radar strategies over an indoor archaeological site: convent of Santo Domingo (Lugo, Spain). Archaeol. Prospect. 2010; 17: 213-22.

[14]. Verdonck L, Vermeulen F, Docter R, Meyer C, Kniess R. 2D and 3D groundpenetrating radar surveys with a modular system: data processing strategies and 
results from archaeological field tests. Near Surface Geophysics 2013; 11(2): 239-52.

331

332

333

334

335

336

337

338

339

340

341

342

343

344

345

346

347 [20]. Zanzi L, Lualdi M, Braun HM, Borisch W, Triltzsch G. An ultra-high frequency

[15]. Lualdi M, Zanzi L, Sosio G. A 3D GPR survey methodology for archaeological applications. Proceedings of the Eleventh International Conference on Ground Penetrating Radar (GPR 2006) June 19-22; Columbus, Ohio, USA.

[16]. Binda L, Lualdi M, Saisi A, Zanzi L. Radar investigation as a complementary tool for the diagnosis of historic masonry buildings, Int. J. of Materials and Structural Integrity 2011; 5(1): 1-25.

[17]. Binda L, Lualdi M, Saisi A. Investigation strategies for the diagnosis of historic structures: On-site tests on Avio Castle, Italy, and Pišece Castle, Slovenia. Can. J. Civil Eng. 2008; 35: 555-66.

[18]. Binda L, Cantini L, Lualdi M, Tedeschi C, Saisi A, Zanzi L. Investigation on structures and materials of the Castle of Avio (Trento, Italy), Adv. Architect. 2005; 20: 599-610.

[19]. Lualdi M, Zanzi L. Testing a safe acquisition procedure for an effective application of GPR to security operations. Proceedings of the Symposium on the Application of Geophysics to Engineering and Environmental Problems (SAGEEP) 2005 Apr. 3-7; Atlanta, USA; p. 613-23.

radar sensor for humanitarian demining tested on different scenarios in $3 \mathrm{D}$ imaging mode. Proc. SPIE 2002 Apr. 15; 4758(1): 240-45. 
350 [21]. Torrione P, Collins LM. Texture Features for Antitank Landmine Detection Using Ground Penetrating Radar. IEEE Transactions on Geoscience and Remote Sensing 2007; 45(7): 2374-82.

[22]. Balanis CA. Antenna Theory: Analysis and Design. 1st ed. New York, US: Wiley \& Sons, 1982.

[23]. Roberts RL, Daniels JJ. Analysis of GPR polarization phenomena. J. Environ. Eng. Geophysics 1996; 1(2): 139-57.

[24]. Fokkema JT, Slob EC, Fokkema E, Beekman S. Material response analysis of georadar reflection data. Near Surface Geophysics 2004; 2(1): 41-7.

[25]. Radzevicius SJ, Daniels DJ. Ground penetrating radar polarization and scattering from cylinders. Journal of Applied Geophysics 2000; 45(2): 111-25.

[26]. Sassen DS, Everett ME, Munster CL. Ecohydrogeophysics at the Edwards Aquifer: insights from polarimetric ground-penetrating radar. Near Surface Geophysics 2009; 7(5): 427-38.

[27]. Tsoflias GP, Van Gestel JP, Stoffa PL, Blankenship DD, Sen M. Vertical fracture detection by exploiting the polarization properties of ground-penetrating radar signals. Geophysics 2004; 69(3): 803-10.

[28]. Van der Kruk J, Zeeman JH, Groenenboom J, Multicomponent imaging of different objects with different strike orientation. Proceedings of the Ninth International Conference on Ground Penetrating Radar 2002 Apr. 29 - May 2; Santa Barbara, USA; p. 150-5. 
371 [29]. Lehmann F, Boerner DE, Holliger K, Green AG. Multicomponent georadar data: Some important implications for data acquisition and processing. Geophysics 2000; 65(5): 1542-52.

[30]. Lualdi M, Lombardi F. Combining Orthogonal Polarization for Full Linear Target Detection with GPR. Forthcoming 2013.

[31]. Lualdi M. True 3D Acquisition using GPR over small areas: A cost effective solution. Proceedings of the Symposium on the Application of Geophysics to Engineering and Environmental Problems (SAGEEP) 2011 Apr. 11; Charleston, South Carolina, US; p. 541-50.

[32]. Zanzi L, Lualdi M. Analysis of Approximations and Aperture Distortion for 3DMigration of Bistatic Radar Data with the Two-Step Approach. EURASIP Journal on Advances in Signal Processing 2010, 2010.

[33]. Pratt WK. Digital Image Processing. 4th ed. New York, US: Wiley \& Sons, 2007.

[34]. Valente M. Seismic performance assessment of a non-ductile RC building retrofitted by steel bracing or fiber-reinforced polymers. Applied Mechanics and Materials 2012; 234: 84-9.

[35]. Valente M. Seismic strengthening of non-ductile R/C structures using infill wall or ductile steel bracing. Advanced Materials Research 2013; 602: 1583-87.

[36]. Birken R, Miller DE, Burns M, Albats P, Casadonte R, Deming R, et al. Efficient large scale underground utility mapping with a multi-channel ground 

USA; p. 186-98.

395 [37]. Böniger U, Tronicke J. On the potential of kinematic GPR surveying using a self-tracking total station: Evaluating system cross-talk and latency. IEEE Transactions on Geoscience and Remote Sensing 2010; 48(10): 3792-98.

398 [38]. Grasmueck M, Viggiano DA. Integration of ground-penetrating radar and laser position sensors for real-time 3-D data fusion. IEEE Transactions on

400 Geoscience and Remote Sensing 2007; 45(1): 130-7.

401 [39]. Groenenboom J, Van Der Kruk J, Zeeman JH. 3D GPR data and the influence of 402 positioning errors on image quality. 63rd EAGE Conference \& Exhibition 2001 June 11-15, Amsterdam, The Netherlands. 